\title{
Vaccines for pandemics
}

\begin{abstract}
The 2009 H1N1 pandemic showed that the egg-based technology used to produce the bulk of flu vaccine is not nimble enough to protect during a pandemic. Charles Schmidt reports on progress in synthetic and recombinant technologies that may provide an answer.
\end{abstract}

Last April, the first reports of a new influenza virus appeared. Health officials in China reported an outbreak of a new avian virus, H7N9, to the World Health Organization (WHO). More than 140 cases were reported within a month, mostly in people working with poultry; 44 died. The DNA sequence was posted by Chinese authorities on March 31, and a week later a panel of synthetic viruses for a potential vaccine had been made by researchers at Novartis Vaccines and Diagnostics in Cambridge, Massachusetts. Combining the ability to transmit sequence data around the world instantaneously with a synthetic process for creating seed strains meant that virus was available for testing before the US Centers for Disease Control (CDC) in Atlanta had even received the viral isolate from China. (It arrived on April 11.)

Novartis' new synthetic influenza virus vaccines will need convincing safety data in humans, however, before regulators give the green light for commercial launch. Meanwhile, incremental gains in efficiency are being introduced into the production scheme by partially or totally replacing the traditional egg-based manufacturing platform. The question is whether any of the new generation of flu vaccines will be ready in time for the next pandemic.

\section{A new generation of vaccines}

Since the 1940s, health officials have tried to contain both seasonal and pandemic flu threats with vaccines manufactured in chicken eggs. Today's manufacturing capacity produces up to 400 million doses a year worldwide, according to the CDC. But time delays in vaccine delivery-particularly risky during pandemic responses-plus questionable efficacy and risks for people with egg allergies have experts increasingly worrying that the egg-based system is outdated and outmoded. "We're holding our breath every year about getting the right vaccine in sufficient amounts at the right time," says Anthony Fauci, director of the National Institute of Allergy and Infectious Diseases (NIAID) in Bethesda, Maryland.

Now flu vaccine manufacturing is going in new directions. Companies are shifting from egg-based manufacturing to cell-based systems to totally synthetic production of viral antigens or whole viruses, using the sequences of viral isolates deposited in public databases. With this approach, there's no waiting for health organizations to come up with the season's seed virus for vaccine manufacturers (Box 1). These next-generation products have already had some success at the regulatory level; last November, the US Food and Drug Administration (FDA) approved the first cell-based vaccine for influenza, Basel-based Novartis' Flucelvax, which is manufactured in dog kidney cells. And

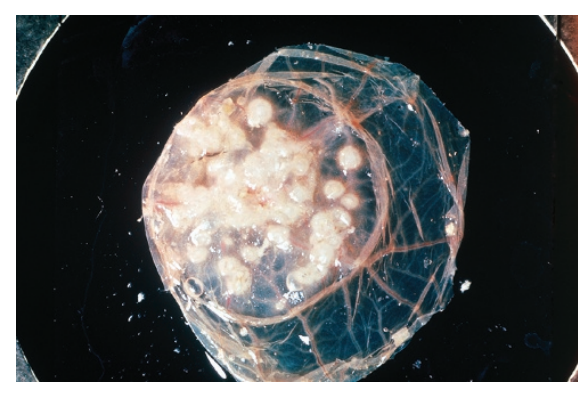

Vaccines growing in egg culture may one day be a thing of the past. (Source: Alamy)
"But we have to go in these new directions," he emphasizes. "We simply can't remain in the current situation; we need to move vaccine development into the twenty-first century."

\section{A new suit for every season}

An RNA virus, influenza is made up of eight segments: a highly conserved six-segment backbone that changes little from one strain to the next, and two antigenic segmentshemagglutinin (HA) and neuraminidase (NA). Two types of influenza account for most human illness; influenza $\mathrm{A}$, with 16 known subtypes (determined by their two antigen sequences) and influenza $B$, with just one subtype. Both influenza A and B are subject to antigenic drift, which means that the antigens accumulate small genetic changes over time. As this occurs, vaccines lose efficacy because newly mutated seasonal strains can evade preexisting immunity. Moreover, influenza A can also undergo antigenic shifts-generally involving more drain January, the FDA approved Flublok, the first completely egg-free vaccine, which is produced in an insect cell line by Protein Sciences, in Meriden, Connecticut. Likewise, two cell-based vaccines have now been approved in Europe (Table 1).

However, these products are not expected to make a sizeable dent in near-term vaccine demand. Novartis plans to bring 30 million vaccine doses to market this year of which only 2 million will be Flucelvax. (The remainder will be the traditional, trivalent, egg-based, inactivated influenza vaccine.) The sales target for Flublok is a mere 150,000 doses. Ruben Donis, associate director for policy, evaluation and preparedness at the CDC's influenza division, cautions that these products face considerable hurdles-until economies of scale lower their prices, the next generation of vaccines will not be competitive with traditional vaccines. And there's no guarantee that alternate manufacturing platforms-faster delivery notwithstanding-will supply more effective vaccines, he says. "No pandemic flu virus comes with a warranty that it will grow well in eggs, cell culture or any other host we want to use," says Donis. "No matter the platform, scientists will have to do their homework when it comes to optimizing the growth of that particular virus." Fauci adds that testing emerging platforms for safety and efficacy will take years. matic sequence changes of at least 30\% - that lead to entirely new subtypes against which humans have virtually no immunity. Should they become broadly transmissible, these subtypes can spawn pandemics. This is what occurred when $\mathrm{H} 2 \mathrm{~N} 2$ replaced $\mathrm{H} 1 \mathrm{~N} 1$ in 1957 (H1N1 reemerged pandemically in 2009, after most immunity against it was lost) or when $\mathrm{H} 3 \mathrm{~N} 2$ replaced $\mathrm{H} 2 \mathrm{~N} 2$ in 1968. In some cases, pandemic viruses jump from animals to humans. H7N9, for instance, is primarily a bird virus with little evidence of human-tohuman transmission. It is believed that $\mathrm{H} 7$ viruses infect only people who come in close contact with live poultry. Still, H7 viruses have in some cases become established in horses and swine, the latter being of particular concern because pigs can act as mixing vessels within which influenza reassorts into more transmissible strains. "If and when these strains gain the ability to spread efficiently from person to person, we would expect to see the next pandemic," says John Treanor, chief of infectious disease at the University of Rochester in Rochester, New York.

Going from the identification of a new strain to the commercial availability of a matched vaccine takes valuable time, the chief holdups being egg adaptation and the need to organize millions of fertilized eggs. Time delays are 


\section{Box 1 Seed strains and bottlenecks}

The current vaccine production enterprise has its roots in a surveillance system for monitoring influenza virus that was established by WHO in 1947. WHO officials collaborate with national health agencies to identify circulating strains in either hemisphere. Once those strains are determined, WHO collaborating laboratories prepare a 'seed virus' for vaccine production. That seed virus is created by allowing the circulating strains to mix and reassort genetically with a master strain known as PR8 designed for optimal yield in chicken eggs. The egg-based system was adopted for several reasons: influenza grows well in chicken eggs, but other co-occurring respiratory pathogens, such as rhinovirus, do not. Egg culturing therefore filters out pathogenic contaminants. Moreover, egg adaptation — or the process by which viruses are genetically manipulated for optimal yield in chicken eggs-ensures that the strains sent out for vaccine production are uniform.

However, egg adaptation has a major downside in that, following the reassortment with PR8, hybrid viruses may no longer adequately match the circulating strains they were initially derived from. This happened last year, when the vaccine for seasonal H3N2 was poorly matched to the circulating virus' H3 antigen, resulting in a loss of efficacy.

especially worrisome during pandemic scares. Donis points out that it took six months to produce a vaccine for the pandemic H1N1 strain after it appeared in April 2009. Illnesses peaked in August and September, he says, but a vaccine against the new strain didn't materialize until October.

In the wake of that pandemic, the President's Council of Advisors on Science and Technology issued a report in August 2010, in which it proposed that replacing egg-based manufacturing with alternative platforms-specifically, cell culture and recombinant technology-could shave weeks off vaccine production schedules ${ }^{1}$.

\section{New platforms stepping up}

Emerging evidence with next-generation vaccines supports that view. Novartis spokesperson Liz Power says that with cell-based production, new batches of Flucelvax can be made in weeks rather than months. Novartis plans to expand production of the vaccine to 50 million seasonal doses at its new \$1-billion manufacturing facility in Holly Springs, North Carolina, which was developed in partnership with the US Department of Health and Human Services. The company is working with FDA to license the facility, which was also designed to provide 150 million doses of pandemic influenza vaccine within six months after a pandemic is declared.

Philip Dormitzer, global head of virology and research at Novartis Vaccines and Diagnostics, adds that making flu virus synthetically saves additional time. This year, Novartis and La Jolla, California-based Synthetic Genomics Vaccines teamed up on a challenge issued by the Biomedical Advanced Research and Development Authority (BARDA; Washington, DC) to create a synthetic flu virus for making vaccine. BARDA didn't give advance notice of the specific virus it wanted made. But coincidentally, the agency selected $\mathrm{H} 7 \mathrm{~N} 9$, a virus that Novartis already had experience with, having made recombinant vaccine against it in 2009 during the pandemic.

To build it, the scientists incorporated synthetic H7N9 HA and NA genes onto a viral

Table 1 The next generation of flu vaccines

\begin{tabular}{|c|c|c|c|}
\hline Company & Product & Technology & $\begin{array}{l}\text { Phase of } \\
\text { development }\end{array}$ \\
\hline Protein Sciences & Flublok & Trivalent vaccine grown in insect cells & Approved \\
\hline Novartis & Flucelvax & Subunit vaccine prepared in MDCK cells & Approved \\
\hline Novartis & Celtura & H1N1 vaccine prepared in MDCK cells & $\begin{array}{l}\text { Approved in } \\
\text { Germany }\end{array}$ \\
\hline Baxter & Celvapan & H1N1 prepared in Vero cells & $\begin{array}{l}\text { Approved in } \\
\text { the EU }\end{array}$ \\
\hline $\begin{array}{l}\text { Mymetics (Epalinges, } \\
\text { Switzerland) }\end{array}$ & $\begin{array}{l}\text { Seasonal flu } \\
\text { vaccine }\end{array}$ & $\begin{array}{l}\text { Reconstituted membrane with } \\
\text { lipopeptide }\end{array}$ & 2 \\
\hline $\begin{array}{l}\text { Immune Targeting System } \\
\text { (London) }\end{array}$ & Flunizyn & Fluorpeptide & 2 \\
\hline $\begin{array}{l}\text { Medicago (Research Triangle } \\
\text { Park, North Carolina }\end{array}$ & $\begin{array}{l}\text { H1N1 VLP } \\
\text { vaccine }\end{array}$ & Virus-like particle & 2 \\
\hline $\begin{array}{l}\text { Vaxinnate } \\
\text { (Cranbury, New Jersey) }\end{array}$ & VAX125 & $\begin{array}{l}\text { HA linked to flagellin for stimulating toll- } \\
\text { like receptors }\end{array}$ & 2 \\
\hline $\begin{array}{l}\text { NovaVax } \\
\text { (Rockville, Maryland) }\end{array}$ & NVAX, H7N9 & $\begin{array}{l}\text { Virus-like particles produced in insect } \\
\text { cells }\end{array}$ & 2 \\
\hline Inovio & FVH1 & Synthetic DNA vaccine & 1 \\
\hline ¡BIO (Newark, Delaware) & $\mathrm{HAC} 1$ & Fusion protein expressed in plants & 1 \\
\hline Vical (San Diego) & Vaxfectin & $\begin{array}{l}\text { Three plasmids encoding two nucleopro- } \\
\text { teins and ion channel protein }\end{array}$ & 1 \\
\hline Astellas/UMN (Tokyo) & ASP7373 & HA protein made in cell culture & 1 \\
\hline $\begin{array}{l}\text { Medigen Biotechnology } \\
\text { (Frederick, Maryland) }\end{array}$ & AT-310 & Whole virus grown in MDCK cells & 1 \\
\hline $\begin{array}{l}\text { Vivaldi Biosciences } \\
\text { (New York) }\end{array}$ & LAIV & Truncated nonstructural protein & Preclinical \\
\hline CureVac & $\begin{array}{l}\text { RNActive } \\
\text { vaccine }\end{array}$ & Self-assembling nanoparticles & Preclinical \\
\hline $\begin{array}{l}\text { Vaccine Research Center } \\
\text { (NIH) }\end{array}$ & HA nanoparticles & mRNA complexed with protamine & Not available \\
\hline
\end{tabular}

backbone variant (PR8x) designed specifically for high-yield virus production in MadinDarby canine kidney cells. This was achieved with a novel method. The genes were assembled in pairs of complementary strands and then melted and reannealed, creating hybrid duplexes in which errors in synthesis would appear as mismatches. The mismatches were corrected with proofreading enzymes. This step alone saved weeks, which would ordinarily have been taken up by resequencing and selecting perfectly correct sequences. "We got the antigenic sequences on Monday and we had a virus by Friday afternoon," Dormitzer says. According to an account of the study published in Science Translational Medicine in $\mathrm{May}^{2}$, the synthetic vaccine achieved the same immunogenic reaction as the natural virus in ferrets. Treanor says that the effort represents a "phenomenal technological achievement with respect to ironing out shortcuts in the reverse genetics arena," although he pointed out synthetic H7N9 isn't actually needed, given that a seed strain that grows well in eggs is already available.

Another approach using recombinant technology is to forego synthesizing the whole 
virus in favor of fabricating only its antigenic sequences. Just this year, for instance, scientists at NIAID linked the gene for ferritin with that of HA of H1N1 to synthesize a fusion protein in mammalian cells that self-assembles into nanoparticles. According to results published in Nature last $\mathrm{May}^{3}$, the nanoparticles were twice as immunogenic in mice as the licensed seasonal influenza vaccine.

Protein Science's Flublok is also based solely on HA sequences (the three currently circulating, H1N1, H3N2 and B) produced in insect cells (Spodoptera frugiperda; army worm) using the baculovirus expression system. "Compared with a traditional vaccine, we get three times more HA per dose," according to Rachael Felberbaum, the company's director of corporate communications. Currently, the vaccine is grown in a 600-liter bioreactor, which will be expanded to 2,500 liters later this year, and then to 18,000 liters at a facility operated by its Japanese partner UMN Pharma, in Minato Mirai. Felberbaum says that each liter of cell culture generates 1,000 doses of a monovalent pandemic and 100 doses of seasonal (trivalent) vaccine. By contrast, Paris-headquartered Sanofi Pasteur-among the world's largest influenza vaccine producers-can process 600,000 eggs per day, each one delivering 1 to 3 doses depending on how well the virus grows in eggs.

\section{Reverse engineering vaccines}

Blue Bell, Pennsylvania-based Inovio Pharmaceuticals develops DNA vaccines, and in the case of flu is using HA sequences alone rather than sequences from the whole virus. Inovio's approach is to assemble a so-called consensus vaccine using an algorithm that aligns shared amino acid sequences found in hundreds of influenza strains and then employing manual identification of the most immunologically dominant and conserved residues based on a priori knowledge. The vaccine is fully synthetic, codon-optimized for expression in human cells and administered by means of electroporation directly into muscle cells. Vaccination requires three injections-a potentially cumbersome process that the company is now trying to address with an upgraded delivery system. Last year, the company reported that its consensus $\mathrm{H} 1 \mathrm{~N} 1$ and $\mathrm{H} 5 \mathrm{~N} 1$ vaccines each elicit protective antibodies in humans, even though they aren't matched to any one strain in particular. In July, the company reported that its consensus $\mathrm{H} 7 \mathrm{~N} 9$ vaccine was $100 \%$ protective in mice given a lethal dose of the virus. Moreover, the vaccine was produced for animal testing in just two weeks, instead of the months that it would have taken to prepare it in eggs. According to Joseph Kim, the company's president and CEO, Inovio is now look- ing for translational funding to test its H7N9 vaccine in humans.

David Weiner, a professor at the University of Pennsylvania Perelman School of Medicine, and chair of Inovio's scientific advisory board, says electroporation results in a transient, but high-level production of immunogenic protein. The sudden HA bolus released from cells upon DNA vaccination generates both B- and T-cell responses, he says, whereas standard influenza vaccines made from killed or attenuated live viruses attract only the former.

Likewise, mRNA-based vaccines for influenza also result in B- and T-cell activation, according to Ingmar Hoerr, CEO with CureVac, in Tubingen, Germany. CureVac has several mRNA-based cancer vaccines (for prostate and non-small cell lung cancer) in clinical trials. Last December, CureVac scientists and their collaborator Lothar Stitz, at the Friedrich-Loeffler-Institut, in Greifswald-Insel Reims, Germany, published a proof-of-concept study in Nature Biotechnology, showing that RNA vaccination protects against flu infection in three animal models ${ }^{4}$. Comprising free and protamine-complexed RNA encoding the HA antigen, the vaccine is applied to the skin, where it is taken up by skin cells, dendritic cells and other cell types, Hoerr says, resulting in HA protein expression and immune system activation. Hoerr says the approach offers an advantage in that, unlike DNA vaccines, RNA vaccines don't enter the nucleus. Instead, they reside in the cytosol, where they generate transient protein expression before being degraded. "It's hard to achieve any control over a DNA vaccine once it integrates with the genome," Hoerr says. "In our view, mRNA vaccines offer a safer alternative. In vaccinated cancer patients, all we see is a bit of redness at the injection site and very high levels of immunogenicity."

\section{Next-geneconomics}

Health officials realized after the H1N1 pandemic of 2009-the first in 41 years-that vaccine production had to speed up to meet potentially explosive demands. The pandemic sparked public investment in alternative platforms. BARDA, for instance, partnered with Novartis on costs associated with the company's Holly Springs facility, and it also supplied Protein Sciences with a \$147-million, fiveyear grant to help pay for the development of Flublok. BARDA has also put money behind programs to improve surveillance and sterility testing.

Support for Inovio's flu program comes chiefly from US and Canadian government sources as well, according to CEO Kim. In 2010, Inovio and its collaborators at the
University of Pennsylvania won a \$3.1-million transformative research award from the Office of the Director at the US National Institutes of Health. This year, the company received a \$3.5-million grant from NIAID to cover ongoing development of its skin electroporation device. The Canadian Institutes of Health Research funds the company's early-stage clinical trials, and as a publicly traded entity, Inovio also relies on internal investor funds to cover its R\&D costs.

But Protein Sciences' Felberbaum adds that the government doesn't support commercial activity. "They get you to the finish line, but then you're on your own," she says. "Our strategy is to start small and then see who will buy." At $\$ 32$ per dose, Flublok will be more expensive than traditional egg-based influenza vaccines, for which the cost per dose to the private sector ranges from $\$ 10$ to $\$ 21$, according to the CDC. Felberbaum says that to justify the added cost, Protein Sciences will market Flublok on the basis of purity. "Being egg-free, it's also antibiotic-free and free of preservatives, and it contains three times the active ingredient," she says. "And as we scale up in production, we expect the vaccine's costs to come down."

Fauci cautions that none of the alternative platforms has proven efficacy in human populations. The true test of vaccine efficacy, he says, will come during seasonal or pandemic outbreaks in the field. And Donis points out that although alternative platforms might offer more flexibility in flu response-in part because they don't require millions of fertile chicken eggs - they don't guarantee scale-up during pandemics. "You need expensive bioreactor systems for cell culturing," he says. "A drug company wouldn't be able to go from producing 2,000 gallons of cell culture to 2 million gallons in a week if they don't have the tanks." Moreover, making vaccines in cells is radically different from making them in eggs, he says, and the more radical the innovation, the more work it takes to satisfy regulatory requirements for safety and efficacy.

\section{Toward a universal vaccine}

Meanwhile, health officials hope to move beyond seasonal or pandemic vaccines toward what Donis describes as the holy grail: universal vaccines that might be protective for years with a single dose. Scientists are exploring various methods for making universal vaccines. Inovio, for instance, claims its consensus approach moves in this direction because the conserved sequences used to create recombinant HA antigen come from hundreds of strains, rather than just one. According to Kim, what the vaccine loses by way of not matching perfectly with any one strain it makes up for 
with protective cross-reactivity against multiple strains. "The virus can't simply mutate its way around the vaccine," he says. "Our approach broadens immune responses against multiple circulating viruses."

But some scientists also say that to create a universal vaccine, research programs need to move away from a predominant focus on the HA head. Shaped like a mushroom, HA antigen comprises a head, which has broad exposure to the immune system, and a less accessible stalk, or stem. The HA head routinely undergoes antigenic drift, but the stem's sequence is conserved among influenza subtypes. Last year, the Center for Infectious Disease Research and Policy (CIDRAP) at the University of Minnesota issued a report in which it claimed that most of the influenza vaccines now in clinical trials around the world were developed to elicit antibodies against the HA head. And that approach, says the CIDRAP's director, Michael Osterholm, forces vaccine producers to keep pace with how the HA head changes every year. "We're just getting caught up in how to make the old vaccines faster," says Osterholm. "We need to think instead about how to make better vaccines." The way to do that, Osterholm says, is to target more conserved regions of the influenza virus, including the HA stalk.

Peter Palese, professor and chair of microbiology at Mount Sinai Hospital in New York, who works on stalk-directed antibodies, agrees. Palese's approach is to trick the immune system, by introducing a vaccine that couples the HA stalk with an exotic head unlike any the immune system has seen before. Anyone who has been immunized or had the flu bears an immunogenic memory of the HA stalk, he explains. And when confronted with an unfamiliar HA head, the immune system's response against the stalk is triggered and amplified.
Palese recently published study results in the Journal of Virology $y^{5}$ showing that vaccines targeting the stalk domain of $\mathrm{H} 3 \mathrm{HA}$ achieved $100 \%$ protection in mice exposed to five influenza clades, including H3, H10, H14, H15 and H7. "But the only way we're really going to know if this works is by testing it in humans," Palese says. "If someone wants to let me try I'll send the vaccine tomorrow."

Donis also agrees that targeting the HA stalk offers possibilities for a universal vaccine. But he questions whether stalk-directed vaccinations can achieve sufficient antibody responses. The stalk's epitopes tend to be obscured from immune surveillance, Donis says, and that lack of access might impair vaccine effectiveness and potency. "You might need a lot more vaccine in the bloodstream to get the job done," he says. "And we don't know how the immune system will tolerate such high levels of stalkdirected antibody."

In addition, recent studies in pigs in which immunization with a vaccine that induced stem-specific antibodies led to more severe pneumonia upon challenge with the flu virusa phenomenon called "antibody-induced disease"-suggests caution should be exercised when adopting a new modality ${ }^{6}$.

Meanwhile, early-stage research in vaccine development still faces substantial regulatory and economic hurdles. Fauci emphasizes that the FDA's job is to ensure safety as much as efficacy, but according to the CIDRAP report, US regulatory processes are designed for "incremental changes to existing vaccines and present a barrier to the development of gamechanging vaccines."

Similarly, European regulatory structures vary by country, and these fragmented policies also interfere with commercial vaccine development, according to Aiswariya
Chidambaram, a senior life sciences analyst with Frost \& Sullivan, based in Chennai, India. A major challenge, Dormitzer adds, comes with adapting infrastructure for pandemic responses; companies that wait until a looming outbreak begins to spread might not produce enough vaccine in time. Raburn Mallory, senior director for clinical development in vaccines and infectious disease with AstraZeneca/MedImmune, in Gaithersburg, Maryland, emphasizes that smaller players in the vaccine market can't easily shift from eggs to alternative platforms. MedImmune produces FluMist, an inhalable attenuated live influenza vaccine made in eggs. According to Mallory, the US government is placing its bets on cell-based systems. "We looked at cell culture, but it's hard to think about switching to an alternative system and go through all that clinical development when the product we have already works," she says.

Still, governments by necessity have a crucial funding role in developing new influenza vaccines, Fauci emphasizes. That's because publicly funded scientists can help to de-risk innovation, he explains, and supply proven technologies that the private sector could find more economically palatable. And as to which of the new platforms will ultimately succeed, Fauci declines to predict. "There are multiple options for how to do this," he says. "What's important is that none of them require that we grow the virus. All we need is the viral sequence."

Charles Schmidt, Portland, Maine

1. Strauss, S. Nat. Biotechnol. 28, 1227-1228 (2010).

2. Dormitzer, P.R. et al. Sci. Transl. Med. 5, 185ra68 (2013).

3. Kanekiyo, M. et al. Nature 499, 102-106 (2013).

4. Petsch, B. et al. Nat. Biotechnol. 30, 1210-1216 (2012).

5. Margine, I. et al. J. Virol. 87, 10435-10446 (2013). 6. Khurana, S. et al. Sci. Transl. Med. 5, 200ra114 (2013). 